\title{
A Large Uterine Leiomyoma with Hypertrophied Omental Vessels: A Case Report
}

\author{
Ayse Nur Aksoy ${ }^{1 *}$, Ebru Bulut Erdem ${ }^{2}$, Gülsah Tanas Sarıkas ${ }^{2}$ and Elçin Telli ${ }^{1}$ \\ ${ }^{1}$ Department of Obstetrics and Gynecology, Erzurum Regional Training and Research Hospital, University of \\ Health Sciences, Erzurum, Turkey \\ ${ }^{2}$ Department of Obstetrics and Gynecology, Nenehatun Hospital, Erzurum, Turkey
}

*Corresponding author: Ayse Nur AKSOY, Department of Obsterics and Gynecology, Erzurum Regional Training and Research Hospital, University of Health Sciences, Erzurum, Turkey, Tel: +905534705222, Fax: +904423172294

\section{Introduction}

Uterine leiomyomas are the most common solid benign uterine neoplasms that arise from the overgrowth of smooth muscle and connective tissue in the uterus. It is estimated that $20-40 \%$ of women of reproductive age have uterine leiomyoma; the prevalence increases during the reproductive age and decreases after menopause $[1,2]$.

Uterine leiomyomas have been classified according to their location: Submucosal, intramural, subserosal. While subserosal uterine leiomyomas are usually asymptomatic; intramural and submucosal uterine leiomyomas are symptomatic. The most common presenting symptoms of leiomyomas are menorrhagia, dysmenorrhea, subfertility, pelvic pain, dyspareunia and pressure symptoms from the bowel and bladder, e.g. constipation, frequency, chronic urinary tract infections $[3,4]$. Clinical examination (medical history, abdominal palpation, speculum examination, bimanual palpation), utrasonographic examination, computed tomography scanning and magnetic resonance imaging are often to diagnose fibroids [5].

In this case report, we aimed to present the surgical management of a postmenopausal patient with a large uterine leiomyoma and omental large dilated blood vessels.

\section{Case Description}

Written informed consent was obtained from the patient for publication of this case report.

A 52-year-old postmenopausal multiparous woman (gravida 8, para 5, aborta 3) presented with a history of lower abdominal pain and distension for about 10 months. The patient's personal history revealed fatigue, loss of appetite, dyspnea and chronic constipation. She had no history of medical illness or surgical procedures. Her last menstrual period was 1 year back and she had no post-menopausal bleeding. But her history ofmenstruation was 7-8 days of the 21-day menstrual cycle. On the other hand, she had not received any hormone replacement therapy and she had no family history of malignancies. Her vital signs were all within normal limits. On physical examination, an abdominopelvic mass with a dullness on percussion - a solid mass was palpated. Abdominal tenderness was not present. Gynecologic examination revealed a large, mobile abdominopelvic mass with a normal external genitalia and uterine cervix. The results of routine laboratory testing including a completeblood count, serum electrolyte levels, tumor markers, tests of liver and renal function and Pap smear were normal except the hemoglobin level, which was 8 $\mathrm{g} / \mathrm{dl}$. Chronic anemia was considered because she had a history of heavy blood loss before menopause.

Abdominal ultrasonography revealed a large solid mass, approximately $20 \mathrm{~cm} \times 15 \mathrm{~cm}$ in size, occupying the abdomen. Ovaries couldn't observed due to solid mass. Magnetic Resonance Imaging of pelvic area revealed an intramural, hypo-intense and homogenous solid uterine mass $200 \mathrm{~mm} \times 150 \mathrm{~mm} \times 150 \mathrm{~mm}$ with

Citation: Aksoy AN, Erdem EB, Sarıkas GT, Telli E (2019) A Large Uterine Leiomyoma with Hypertrophied Omental Vessels: A Case Report. Obstet Gynecol Cases Rev 6:143. doi.org/10.23937/23779004/1410143

Accepted: March 27, 2019: Published: March 29, 2019

Copyright: (c) 2019 Aksoy AN, et al. This is an open-access article distributed under the terms of the Creative Commons Attribution License, which permits unrestricted use, distribution, and reproduction in any medium, provided the original author and source are credited. 

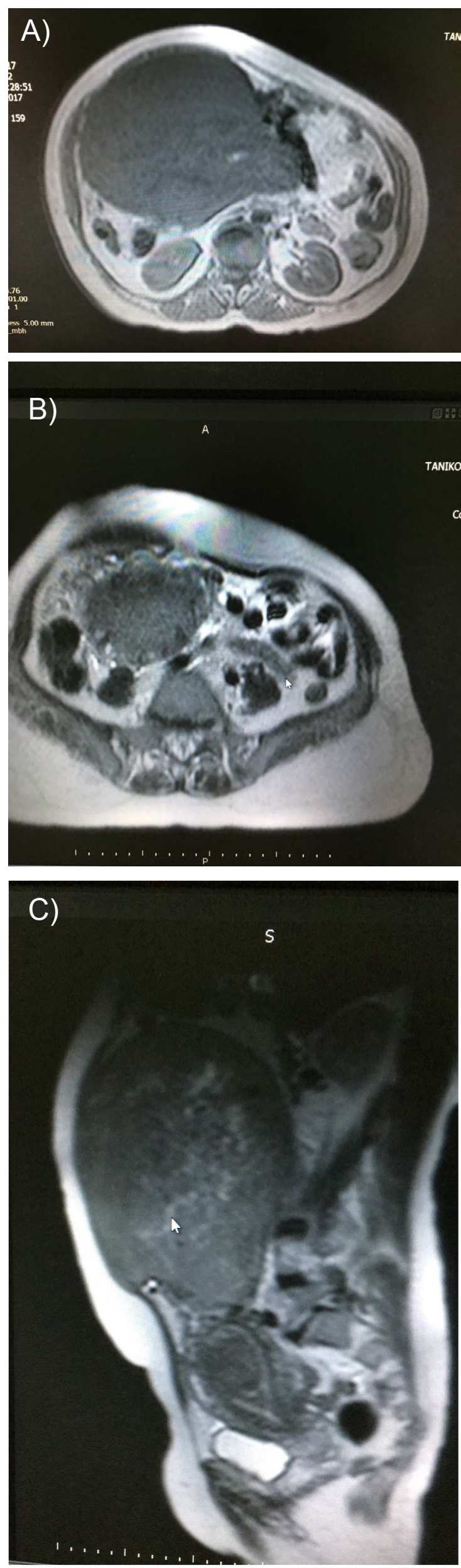

Figure 1: a,b,c) Images of MRI in sagittal and axial sections showing the uterine fibroid mass.

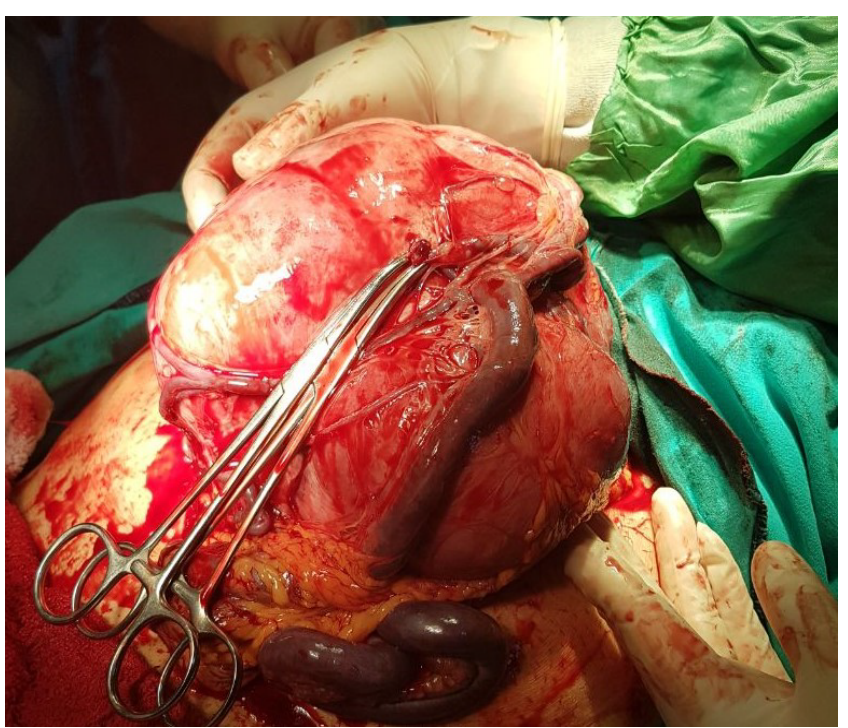

Figure 2: Intraoperative appearance of uterus with large leiomyoma and omental large dilated blood vessels.

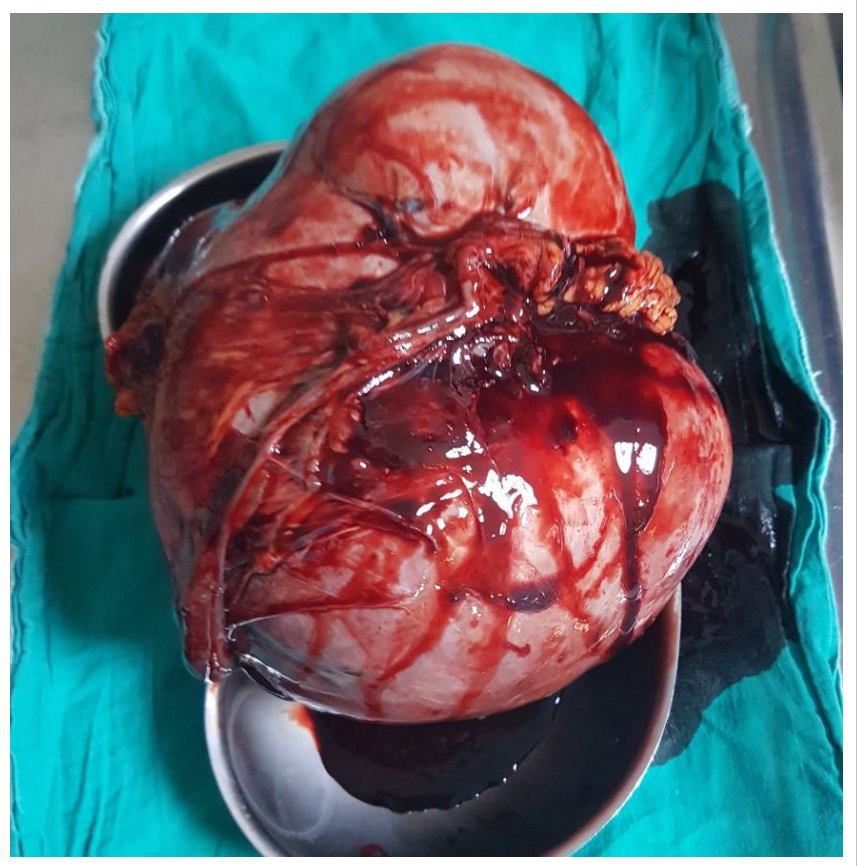

Figure 3: Post-operative specimen showing the uterus with large leiomyoma.

omental large dilated blood vessels measuring upto 5 $\mathrm{mm}$ in diameter (Figure 1a, Figure $1 \mathrm{~b}$ and Figure 1c). Preoperatively, uterine sarcoma was suspected. However, the mass was no fixed, there was no features suggestive of sarcoma (mixed echogenic and poor echogenic parts) ultrasonographically. Also, Magnetic Resonance Imaging findings had no necrosis, hemorrhage and irregular or ill-defined margins. A possible large leiomyoma was suspected and the patient underwent laparotomy. Two units of packed red blood cells were transfused preoperatively. A vertical, midline incision was made $1 \mathrm{~cm}$ below the umbilicus extending down to $2 \mathrm{~cm}$ above the pubic symphysis. At laparotomy, an enlarged intramural leiomyoma arising from uterus that filled the entire lower abdomen with omental large dilated blood ves- 
sels was observed (Figure 2). Peritoneal washing sample was collected. A total abdominal hysterectomy with bilateral salpingo-oophorectomy was performed. After obtaining hemostasis, a hemovac drain was packed into the pelvis to drain any blood for the first 24 hours postoperatively. The drain was removed on the first postoperative day, and she was discharged on the third postoperative day in excellent condition. Low molecular weight heparin (Clexane ${ }^{\circledR}$, Aventis Intercontinental, France) was used for thromboprophylaxi. Ceftriaxone (Rocephine ${ }^{\circledR}$, Roche) was applied $1 \mathrm{~g}$ twice a day. Gross pathologic examination revealed a $20 \mathrm{~cm} \times 20 \mathrm{~cm} \times 15$ $\mathrm{cm}$, 3600-g intramural, myomatous, cellular leiomyoma and bilateral ovaries and tubes were normal (Figure 3). Peritoneal washing showed no malignant cells. Histologic signs of malignancy were not found. The final diagnosis was a intramural uterine leiomyoma with degenerative changes.

\section{Discussion}

Uterine fibroid tumors are the most common benign neoplasms of the female reproductive tract. Unfortunately, the clinical features of benign leiomyomas and uterine sarcomas are often similar. Uterine sarcomas are most commonly diagnosed histologically after resection of uterine tissue (myomectomy or hysterectomy). UItrasonography is preferred for the initial evaluation, because it is the least invasive and cost-effective technical. Expectant management, surgical management, medical management or uterine artery embolization are used in the management of uterine fibroids. The choice of treatment depends on the patient's age, type and severity of symptoms, suspicion of malignancy, desire for future fertility and proximity to menopause. A surgical approach is most frequently preferred for management of large uterine leiomyomas. This case represents a successful surgical management of a postmenopausal patient with large uterine leiomyoma.

Many epidemiologic factors including reproductive factors, age, race, heritage, sex hormones, obesity, lifestyle, environmental influences and infection were reported associated with the development of uterine fibroids [1]. The most common symptoms in uterine fibroids are pelvic pain, abnormal bleeding, and tumor bulk-related symptoms such as voiding problems, fatigue, loss of appetite, dyspnea and chronic constipation, On the other hand, patients may have no symptoms. Indications surgical treatment of uterine leiomyomas are the following: Menorrhagia not responding to medical therapy, suspicion of malignancy, unexplained infertility, fibroids growing after menopause, the presence of symptoms that have a negative effect on daily life [3]. Myomectomy or hysterectomy may be done according to the woman's choice, and her desire for future child bearing in premenopausal women. In post-menopausal women with symptomatic fibroids, hysterectomy rather than myomectomy is preferred due to the risk of malignant changes in the fibroids. Transverse or vertical incision of the abdominal wall may be planned for operation. According to the ACOG recommendation, oophorectomy should be considered at the time of hysterectomy in postmenopausal women [6]. Mülayim B [7] presented a case treated with a total abdominal hysterectomy with bilateral salpingo-oophorectomy due to a large uterine leiomyomas in a 48-year-old multiparous woman without complaints. In another case, Yavuz, et al. [8] reported a 47-year-old premenopausal woman with giant uterine leiomyoma weighing 9.8 kilograms. They performed laparotomy, total hysterectomy and bilateral salpingo-ooferectomy for this patient. Similar to these cases we reported a 52-year-old woman who presented with fatigue, loss of appetite, dyspnea and chronic constipation and investigations indicated a possible diagnosis of a large uterine leiomyoma with omental large dilated blood vessels. She was treated a total abdominal hysterectomy with bilateral salpingo-oophorectomy and she was discharged on the third postoperative day in excellent condition.

Large uterine leiomyomas with omental large dilated blood vessels are unusual cases. Careful perioperative management and multidisciplinary patient care are essential to prevent morbidity and mortality.

\section{Authors' Contribution}

A.N.A., E.B.E.: Conception and design, drafting of the manuscript. G.T.S., E.T.: Conception and design, critical revision of the manuscript.

\section{References}

1. Sparic R, Mirkovic L, Malvasi A, Tinelli A (2016) Epidemiology of uterine myomas: A review. Int J Fertil Steril 9: 424-435.

2. Okolo S (2008) Incidence, aetiology and epidemiology of uterine fibroids. Best Pract Res Clin Obstet Gynaecol 22: 571-588.

3. Levy BS (2008) Modern management of uterine fibroids. Acta Obstet Gynecol 87: 812-823.

4. Evans P, Brunsell S (2007) Uterine fibroid tumors: Diagnosis and treatment. Am Fam Physician 75: 1503-1508.

5. Parker WH (2007) Etiology, symptomatology, and diagnosis of uterine myomas. Fertil Steril 87: 725-736.

6. American College of Obstetricians and Gynecologists (2008) Clinical management guidelines for obstetrician-gynecologists. ACOG Practice Bulletin 89 Washington, DC, 231-241.

7. Mülayim B (2015) Unaware of a large leiomyoma: A case report with respect to unusual symptoms of large leiomyomas. Ann Med Surg (Lond) 4: 431-433.

8. Yavuz A, Astepe B, Terzi H, Kale A (2015) Management of uterine giant myoma: Case report. Sakaryamj 5: 34-38. 